Mathematical Modelling and Analysis

Volume 20 Number 3, May 2015, 311-328

http://dx.doi.org/10.3846/13926292.2015.1048756

(c) Vilnius Gediminas Technical University, 2015
Publisher: Taylor\&Francis and VGTU

http://www.tandfonline.com/TMMA

ISSN: $1392-6292$

eISSN: 1648-3510

\title{
On Some Generalization of Smoothing Problems*
}

\section{Svetlana Asmuss ${ }^{a, c}$ and Natalja Budkina ${ }^{a, b}$}

${ }^{a}$ University of Latvia

Zellu street 8, LV-1002 Riga, Latvia

${ }^{b}$ Riga Technical University

Meza street 1/4, LV-1048 Riga, Latvia

${ }^{c}$ Institute of Mathematics and Computer Science of University of Latvia

Rainis blvd. 29, LV-1459 Riga, Latvia

E-mail: svetlana.asmuss@lu.lv

E-mail(corresp.): natalja.budkina@rtu.lv

Received October 30, 2014; revised March 30, 2015; published online May 15, 2015

\begin{abstract}
The paper deals with the generalized smoothing problem in abstract Hilbert spaces. This generalized problem involves particular cases such as the interpolating problem, the smoothing problem with weights, the smoothing problem with obstacles, the problem on splines in convex sets and others. The theorem on the existence and characterization of a solution of the generalized problem is proved. It is shown how the theorem gives already known theorems in special cases as well as some new results.
\end{abstract}

Keywords: interpolating splines, smoothing splines, mixed splines, splines in convex sets.

AMS Subject Classification: 65D07; 41A15; 41A29.

\section{Introduction}

The connection between variational problems and the theory of splines originates from the paper by J.C. Holladay [8]. An essential step in the development of the theory of splines was the generalization of the notion of a spline in abstract Hilbert spaces, which was obtained by applying methods of functional analysis: the interpolating and smoothing problems were investigated by P.M. Anselone, P.J. Laurent [1]; the problem on splines in a convex set was formulated and the conditions of existence and characterization of a solution of this problem were obtained by M. Attea $[5,6]$. The first systematic approach to the smoothing problem using operators in abstract Hilbert spaces was expounded

* This work is partially supported by the European Social Fund within the project 2013/0024/1DP/1.1.1.2.0/13/APIA/VIAA/045. 
in the monograph by P.J. Laurent [15]. In the framework of this theory (concerning the variational theory of splines in Hilbert spaces we refer to $[7,15,19]$ ) the necessary and sufficient conditions for existence and uniqueness of interpolating and smoothing splines as solutions of variational problems were obtained, the splines in a convex set were investigated in a more general case. This general point of view promoted the study of known problems for spline-functions as well as was helpful for setting and solving new problems. Now it is a usual practice to consider splines as solutions of some optimization (interpolating or smoothing) problems (see, e.g., $[9,11,12,14,16,17,18]$ and references to research papers therein).

There are several works, where some generalizations of interpolating and smoothing variational splines were considered by combining interpolating and smoothing conditions. Mixed interpolating-smoothing problems are studied in [7]. P. Oja and E. Leetma $[16,17,18]$ have investigated smoothing problems with obstacles and weights with a generalization for the case of mixed interpolating-smoothing conditions in Bepo Levi space. In general, the variational theory of splines in the case, when instead of Hilbert spaces semi-Hilbert spaces are considered, is developed in [2]. In the similar framework A. Kouibia and M. Pasadas (see $[9,10,11,12,13,14]$ ) by specifying the spaces and operators have considered variational interpolating and smoothing splines including those in convex sets. These authors have considered different optimization problems where the objective functional is composed by the classical smoothness measure and other terms, which present some different conditions, as, for example, fairness conditions, for specified spaces of solution. Variational interpolating splines corresponding to such objective functional are considered in [11], smoothing problems with weights for objective functionals with some additional terms are studied in $[9,10,12,14]$. A. Kouibia and M. Pasadas have considered also the problem on splines in convex sets in oder to provide, for example, monotonicity constraints for interpolating spline [13]. In this paper we suggest some generalization of smoothing problems on splines in convex sets, which is new in comparison with all approaches mentioned above.

The paper deals with generalized smoothing problem with weights

$$
\|T x\|^{2}+\|\mathbf{R}(A x-\boldsymbol{v})\|^{2} \longrightarrow \min _{x \in B^{-1}(C)},
$$

where $T: X \rightarrow Y, A: X \rightarrow \mathbb{R}^{n}$ and $B: X \rightarrow \mathbb{R}^{m}$ are linear continuous operators, $X$ and $Y$ are Hilbert spaces, $\boldsymbol{v} \in \mathbb{R}^{n}, \mathbf{R}=\operatorname{diag}\left(\sqrt{\rho_{i}}\right)_{i=1, \ldots, n}$ is a diagonal matrix with parameters $\rho_{i} \geq 0, i=1, \ldots, n$, and $C \subset \mathbb{R}^{m}$ is a closed convex set.

This problem generalizes:

- the interpolating problem,

- the smoothing problem with weights,

- the smoothing problem with obstacles,

- the problem on splines in convex sets. 
The classic formulations of these special cases are given in Section "Preliminaries". Section "Generalized smoothing problem" is devoted to the generalized problem and contains the theorem on the existence and characterization of a solution of it. In the next section we consider special cases of the generalized theorem. Firstly, we show how the theorem gives already known results for the interpolating problem, the smoothing problem with weights, the smoothing problem with obstacles, the mixed smoothing-interpolating problem and the problem on splines in convex sets. Secondly, we consider some new special cases of the theorem. The last section illustrates our approaches with some numerical examples.

This research is closely related to our previous papers [3,4] about particular cases of the generalized problem where the spaces and operators were specified. The main aim of the present paper is to provide the general approach to all types of particular problems mentioned above and in this framework to prove the general theorem on the existence and characterization of a solution.

\section{Preliminaries}

Let $X, Y$ be Hilbert spaces and assume that linear operators $T: X \rightarrow Y$, $A: X \rightarrow \mathbb{R}^{n}$ are continuous, $A(X)$ and $T(X)$ are closed. Operators $T$ and $A$ define the corresponding space of splines

$$
S(T, A)=\left\{s \in X:\langle T s, T x\rangle_{Y}=0 \text { for all } x \in \operatorname{ker} A\right\} .
$$

Here and in the sequel $\langle,\rangle_{Y}$ is a scalar product in $Y$ (in the sequel in the article the index of scalar product will not be shown when it is clear from the context), ker $A$ is the kernel of operator $A$.

It is known (e.g. [19]) that an element $s \in X$ is the spline from $S(T, A)$ if and only if there exists such vector $\boldsymbol{\alpha} \in \mathbb{R}^{n}$ that

$$
T^{*} T s=A^{*} \boldsymbol{\alpha} .
$$

For given parameters $\rho_{i}>0, i=1, \ldots, n$, matrix $\mathbf{R}=\operatorname{diag}\left(\sqrt{\rho_{i}}\right)_{i=1, \ldots, n}$, closed convex set $C \subset \mathbb{R}^{n}$ and vector $\boldsymbol{u} \in \mathbb{R}^{n}$ we consider three conditional minimization problems.

Problem 1 [The interpolating problem].

$$
\|T x\| \longrightarrow \min _{\substack{x \in X \\ A x=\boldsymbol{u}}} .
$$

A solution of Problem 1 (if it exists) is a spline from $S(T, A)$ called the interpolating spline for $\boldsymbol{u}$ (see, e.g., $[15,19]$ ).

Problem 2 [The problem on splines in a convex set].

$$
\|T x\| \longrightarrow \min _{x \in A^{-1}(C)} .
$$

A solution of Problem 2 (if it exists) is a spline from $S(T, A)$ called the spline in a convex set (see, e.g., $[15,19])$. 
Problem 3 [The smoothing problem with weights].

$$
\|T x\|^{2}+\|\mathbf{R}(A x-\boldsymbol{u})\|^{2} \longrightarrow \min _{x \in X}
$$

A solution of Problem 3 (if it exists) is a spline from $S(T, A)$ called the smoothing spline (see, e.g., $[15,19])$.

Now let $B: X \rightarrow \mathbb{R}^{m}$ be a linear continuous operator and $C \subset \mathbb{R}^{m}$ be a closed convex. As the first attempt of generalization of Problems 1 and 3 we consider (see [7]) the following problem.

Problem 4 [The mixed interpolating-smoothing problem].

$$
\|T x\|^{2}+\left\|\mathbf{R}^{2}(A x-\boldsymbol{u})\right\|^{2} \longrightarrow \min _{\substack{x \in X \\ B x=\boldsymbol{v}}},
$$

where the restriction given by operator $B$ describes interpolating conditions and the smoothing functional is defined by operators $T$ and $A$. A solution of this problem (if it exists) is a spline from the space $S(T,(A, B))$ (see, e.g., [7]). Here

$$
(A, B): X \rightarrow \mathbb{R}^{n+m}, \quad \text { t.i. } \quad(A, B) x=(A x, B x) .
$$

Taking into account

$$
(A, B)^{*}(\boldsymbol{\alpha}, \boldsymbol{\beta})=A^{*} \boldsymbol{\alpha}+B^{*} \boldsymbol{\beta},
$$

we obtain that an element $s \in X$ is a spline from $S(T,(A, B))$ if and only if there exist such vectors $\boldsymbol{\alpha} \in \mathbb{R}^{n}$ and $\boldsymbol{\beta} \in \mathbb{R}^{m}$ that

$$
T^{*} T s=A^{*} \boldsymbol{\alpha}+B^{*} \boldsymbol{\beta} .
$$

\section{Generalized Smoothing Problem}

Let $X, Y$ be Hilbert spaces and assume that linear operators

$$
T: X \rightarrow Y, \quad A: X \rightarrow \mathbb{R}^{n} \quad \text { and } \quad B: X \rightarrow \mathbb{R}^{m}
$$

are continuous,

$$
T(X)=Y, \quad A(X)=\mathbb{R}^{n}, \quad B(X)=\mathbb{R}^{m} .
$$

Let parameters $\rho_{i} \geq 0, i=1, \ldots, n$, matrix $\mathbf{R}=\operatorname{diag}\left(\sqrt{\rho_{i}}\right)_{i=1, \ldots, n}$, vector $\boldsymbol{v} \in \mathbb{R}^{n}$ and convex closed set $C \subset \mathbb{R}^{m}$ be given.

Our aim is to consider and investigate a generalized smoothing problem with weights in a convex set, which involves all previous problems (Problems 1, 2, 3 and 4) as particular cases of its statement.

Problem 5 [The generalized smoothing problem].

$$
\|T x\|^{2}+\|\mathbf{R}(A x-\boldsymbol{v})\|^{2} \longrightarrow \min _{x \in B^{-1}(C)} .
$$


We formulate the following theorem on the existence and characterization of a solution of Problem 5 in a way which gives a possibility to obtain this solution by using characterization formulas for splines from $S(T, A)$ and $S(T, B)$. It simplifies the use of this result.

Theorem 1. Under the assumptions that

$\operatorname{ker} T+\operatorname{ker} \mathbf{R} A \quad$ and $\quad \operatorname{ker} T \cap \operatorname{ker} \mathbf{R} A+B^{-1}(C)$ are closed,

a solution of Problem 5 exists. An element $s \in B^{-1}(C)$ is a solution of this problem if and only if there exist vectors $\boldsymbol{\alpha} \in \mathbb{R}^{n}$ and $\boldsymbol{\beta} \in \mathbb{R}^{m}$ such that

$$
T^{*} T s=A^{*} \boldsymbol{\alpha}+B^{*} \boldsymbol{\beta}
$$

and

$$
\left\langle\boldsymbol{\alpha}+\mathbf{R}^{2}(A s-\boldsymbol{v}), \boldsymbol{d}-A s\right\rangle+\langle\boldsymbol{\beta}, \boldsymbol{c}-B s\rangle \geq 0
$$

for all $\boldsymbol{c} \in C$ and $\boldsymbol{d} \in A\left(B^{-1}(\boldsymbol{c})\right)$.

Proof. By the pair of operators $T$ and $A$ we introduce the operator $L$ :

$$
L x=(T x, \mathbf{R} A x), \quad x \in X,
$$

taking values in the space $V=Y \times \mathbb{R}^{n}$ with the scalar product

$$
\left\langle\left(y^{1}, \boldsymbol{z}^{1}\right),\left(y^{2}, \boldsymbol{z}^{2}\right)\right\rangle_{V}=\left\langle y^{1}, y^{2}\right\rangle_{Y}+\left\langle\boldsymbol{z}^{1}, \boldsymbol{z}^{2}\right\rangle_{\mathbb{R}^{n}}
$$

and the norm

$$
\|(y, \boldsymbol{z})\|_{V}=\sqrt{\|y\|_{Y}^{2}+\|\boldsymbol{z}\|_{\mathbb{R}^{n}}^{2}}
$$

We introduce the vector $\boldsymbol{e}=\left(\theta_{Y}, \mathbf{R} \boldsymbol{v}\right) \in V$ and rewrite Problem 5 in the form

$$
\|L x-e\|_{V} \longrightarrow \min _{x \in B^{-1}(C)}
$$

To prove the existence of a solution of this problem we check that $L\left(B^{-1}(C)\right)$ is closed. At first we show the closeness of $L(X)$, which is equivalent to the closeness of $L^{*}(V)$. The equality

$$
\langle L x,(y, \boldsymbol{z})\rangle_{V}=\langle T x, y\rangle_{Y}+\langle\mathbf{R} A x, \boldsymbol{z}\rangle_{\mathbb{R}^{n}}
$$

implies

$$
L^{*}(V)=T^{*}(Y)+(\mathbf{R} A)^{*}\left(\mathbb{R}^{n}\right)=\overline{T^{*}(Y)}+\overline{(\mathbf{R} A)^{*}\left(\mathbb{R}^{n}\right)}=(\operatorname{ker} T)^{\perp}+(\operatorname{ker} \mathbf{R} A)^{\perp} .
$$

Taking into account that $\operatorname{ker} T+\operatorname{ker} \mathbf{R} A$ is closed, we obtain that $(\operatorname{ker} T)^{\perp}+$ $(\operatorname{ker} \mathbf{R} A)^{\perp}$ is closed also. Therefore $L^{*}(V)$ and also $L(X)$ are closed.

We note that $\operatorname{ker} L=\operatorname{ker} T \cap \operatorname{ker} \mathbf{R} A$ and consider operator $L^{\perp}$ which is obtained as the restriction of $L$ on $(\operatorname{ker} L)^{\perp}$. This operator is a bijection between 
$(\text { ker } L)^{\perp}$ and closed subspace $L(X)$. We denote the inverse continuous linear operator for $L^{\perp}$ by $D$. Now, taking into account

$$
\begin{aligned}
L\left(B^{-1}(C)\right) & =L\left(B^{-1}(C)+\operatorname{ker} L\right)=L\left(\left(B^{-1}(C)+\operatorname{ker} L\right) \cap(\operatorname{ker} L)^{\perp}\right) \\
& =D^{-1}\left(\left(B^{-1}(C)+\operatorname{ker} L\right) \cap(\operatorname{ker} L)^{\perp}\right),
\end{aligned}
$$

we get that $L\left(B^{-1}(C)\right)$ is closed. The existence of a solution of Problem 5 follows from the existence of the element of the minimal norm in a convex closed set.

To prove the characterization of a solution of Problem 5, we denote it by $s$. So $s \in B^{-1}(C)$ and for any $x \in B^{-1}(C)$ the inequality

$$
\|L x-e\|_{V} \geq\|L s-e\|_{V}
$$

holds. Taking into account that $B s \in C$, we take arbitrary $\boldsymbol{c} \in C$ and consider $\boldsymbol{c}-B s$. There exists such $f \in X$ that $B f=\boldsymbol{c}-B s$. We denote $\boldsymbol{c}_{\lambda}=B(s+\lambda f)$ for $\lambda \in[0 ; 1]$. Obviously $\boldsymbol{c}_{\lambda} \in C$ and $s+\lambda f \in B^{-1}(C)$. Now by (3.2) for any $\lambda \in[0 ; 1]$ we get

$$
\|L(s+\lambda f)-e\|_{V}^{2} \geq\|L s-e\|_{V}^{2}
$$

SO

$$
\begin{aligned}
\|L s-\boldsymbol{e}+\lambda L f\|_{V}^{2}-\|L s-\boldsymbol{e}\|_{V}^{2} & =2 \lambda\langle L s-\boldsymbol{e}, L f\rangle_{V}+\lambda^{2}\langle L f, L f\rangle_{V} \\
& =2 \lambda\langle L s-\boldsymbol{e}, L f\rangle_{V}+\lambda^{2}\|L f\|_{V}^{2} \\
& =\lambda\left(2\langle L s-\boldsymbol{e}, L f\rangle_{V}+\lambda\|L f\|_{V}^{2}\right) \geq 0 .
\end{aligned}
$$

Therefore for any $\lambda \in[0 ; 1]$ we get $2\langle L s-\boldsymbol{e}, L f\rangle_{V}+\lambda\|L f\|_{V}^{2} \geq 0$. It means that $\langle L s-\boldsymbol{e}, L f\rangle_{V} \geq 0$ or

$$
\langle(T s, \mathbf{R}(A s-\boldsymbol{v})),(T f, \mathbf{R} A f)\rangle_{V} \geq 0
$$

or

$$
\langle T s, T f\rangle_{Y}+\langle\mathbf{R}(A s-\boldsymbol{v}), \mathbf{R} A f\rangle_{\mathbb{R}^{n}} \geq 0 .
$$

We note that for all $f \in \operatorname{ker} B$ the inequality (3.3) holds because in the special case we can take $\boldsymbol{c}=B s$.

Now we prove that $s \in S(T,(A, B))$. It is easy to see by (3.3) that for $f \in \operatorname{ker} A \cap \operatorname{ker} B$

$$
\langle T s, T f\rangle_{Y} \geq 0 \quad \text { and } \quad\langle T s, T(-f)\rangle_{Y} \geq 0 .
$$

Therefore for any $f \in \operatorname{ker} A \cap \operatorname{ker} B\langle T s, T f\rangle_{Y}=0$, i.e. $s \in S(T,(A, B))$.

The definition of the space of splines $S(T,(A, B))$ implies that $s \in$ $S(T,(A, B))$ if and only if there exist vectors $\boldsymbol{\alpha} \in \mathbb{R}^{n}$ and $\boldsymbol{\beta} \in \mathbb{R}^{m}$ such that

$$
T^{*} T s=A^{*} \boldsymbol{\alpha}+B^{*} \boldsymbol{\beta} .
$$

From this equality by using (3.3) we get

$$
\left\langle A^{*} \boldsymbol{\alpha}, f\right\rangle_{X}+\left\langle B^{*} \boldsymbol{\beta}, f\right\rangle_{X}+\langle\mathbf{R}(A s-\boldsymbol{v}), \mathbf{R} A f\rangle_{\mathbb{R}^{n}} \geq 0
$$


or

$$
\langle\boldsymbol{\alpha}, A f\rangle_{\mathbb{R}^{n}}+\langle\boldsymbol{\beta}, B f\rangle_{\mathbb{R}^{m}}+\left\langle\mathbf{R}^{2}(A s-\boldsymbol{v}), A f\right\rangle_{\mathbb{R}^{n}} \geq 0 .
$$

Taking into account that by our denotation $B f=\boldsymbol{c}-B s$ we rewrite (3.4) as

$$
\left\langle\boldsymbol{\alpha}+\mathbf{R}^{2}(A s-\boldsymbol{v}), \boldsymbol{d}-A s\right\rangle_{\mathbb{R}^{n}}+\langle\boldsymbol{\beta}, \boldsymbol{c}-B s\rangle_{\mathbb{R}^{m}} \geq 0,
$$

where $\boldsymbol{d}=A f+A s$. Thereby we proved that (3.5) is true for all $\boldsymbol{c} \in C$ and $\boldsymbol{d} \in A\left(B^{-1}(\boldsymbol{c})\right)$.

To prove the second part of our theorem, we assume that for $s \in B^{-1}(C)$ there exist vectors $\boldsymbol{\alpha} \in \mathbb{R}^{n}$ and $\boldsymbol{\beta} \in \mathbb{R}^{m}$ such that

$$
T^{*} T s=A^{*} \boldsymbol{\alpha}+B^{*} \boldsymbol{\beta},
$$

i.e. $s \in S(T,(A, B))$, and for all $\boldsymbol{c} \in C$, and $\boldsymbol{d} \in A\left(B^{-1}(\boldsymbol{c})\right)$ it holds

$$
\left\langle\boldsymbol{\alpha}+\mathbf{R}^{2}(A s-\boldsymbol{v}), \boldsymbol{d}-A s\right\rangle_{\mathbb{R}^{n}}+\langle\boldsymbol{\beta}, \boldsymbol{c}-B s\rangle_{\mathbb{R}^{m}} \geq 0 .
$$

To prove that $s$ is a solution of Problem 5 we should show that for any $x$ from $B^{-1}(C)$ the inequality

$$
\|L x-e\|_{V} \geq\|L s-e\|_{V}
$$

holds. We rewrite

$$
\begin{aligned}
\|L x-e\|_{V}^{2}-\|L s-\boldsymbol{e}\|_{V}^{2} & =\langle L x-\boldsymbol{e}, L x-\boldsymbol{e}\rangle_{V}-\langle L s-\boldsymbol{e}, L s-\boldsymbol{e}\rangle_{V} \\
& =\langle L x-L s, L x-L s\rangle_{V}+2\langle L s-\boldsymbol{e}, L x-L s\rangle_{V} \\
& =\|L x-L s\|_{V}^{2}+2\langle L s-\boldsymbol{e}, L x-L s\rangle_{V} .
\end{aligned}
$$

To prove that the last expression is nonnegative we analyze the last term of this expression. We rewrite it as

$$
\begin{aligned}
\langle L s- & \boldsymbol{e}, L x-L s\rangle_{V}=\langle T s, T(x-s)\rangle_{Y}+\langle\mathbf{R}(A s-\boldsymbol{v}), \mathbf{R}(A x-A s)\rangle_{\mathbb{R}^{n}} \\
& =\left\langle T^{*} T s,(x-s)\right\rangle_{X}+\left\langle\mathbf{R}^{2}(A s-\boldsymbol{v}), A x-A s\right\rangle_{\mathbb{R}^{n}} \\
& =\left\langle A^{*} \boldsymbol{\alpha},(x-s)\right\rangle_{X}+\left\langle B^{*} \boldsymbol{\beta},(x-s)\right\rangle_{X}+\left\langle\mathbf{R}^{2}(A s-\boldsymbol{v}), A x-A s\right\rangle_{\mathbb{R}^{n}} \\
& =\left\langle\boldsymbol{\alpha}+\mathbf{R}^{2}(A s-\boldsymbol{v}), A x-A s\right\rangle_{\mathbb{R}^{n}}+\langle\boldsymbol{\beta}, B x-B s\rangle_{\mathbb{R}^{m}} .
\end{aligned}
$$

For any $x \in B^{-1}(C)$ by denotation $\boldsymbol{c}=B x$ we have $x \in B^{-1}(\boldsymbol{c})$ and therefore $A x \in A\left(B^{-1}(\boldsymbol{c})\right)$. We denote this vector $A x$ by $\boldsymbol{d}$ and rewrite (3.6) as

$$
\left\langle\boldsymbol{\alpha}+\mathbf{R}^{2}(A s-\boldsymbol{v}), \boldsymbol{d}-A s\right\rangle_{\mathbb{R}^{n}}+\langle\boldsymbol{\beta}, \boldsymbol{c}-B s\rangle_{\mathbb{R}^{m}} .
$$

The last expression is nonnegative for all $\boldsymbol{c} \in C$ and $\boldsymbol{d} \in A\left(B^{-1}(\boldsymbol{c})\right)$ by the conditions of the theorem. Therefore

$$
\|L x-e\|_{V}^{2}-\|L s-e\|_{V}^{2} \geq 0 .
$$

The characterization theorem implies that a solution of Problem 5 is a spline from the space of splines $S(T,(A, B))$. An element $s \in X$ is a spline of $S(T,(A, B))$ if and only if there exist such vectors $\boldsymbol{\alpha} \in \mathbb{R}^{n}$ and $\boldsymbol{\beta} \in \mathbb{R}^{m}$ that

$$
T^{*} T s=A^{*} \boldsymbol{\alpha}+B^{*} \boldsymbol{\beta} .
$$

Taking into account that 
- $s_{1} \in S(T, A)$ if and only if there exists such $\boldsymbol{\alpha} \in \mathbb{R}^{n}$ that $T^{*} T s_{1}=A^{*} \boldsymbol{\alpha}$,

- $s_{2} \in S(T, B)$ if and only if there exists such $\boldsymbol{\beta} \in \mathbb{R}^{m}$ that $T^{*} T s_{2}=B^{*} \boldsymbol{\beta}$,

we obtain $S(T, A)+S(T, B) \subset S(T,(A, B))$, but in general the spaces $S(T, A)+$ $S(T, B)$ and $S(T,(A, B))$ are not equal. At the same time this approach gives a possibility to write the formula for splines from $S(T,(A, B))$ by using known formulas for splines from each space: $S(T, A)$ and $S(T, B)$.

To illustrate this fact we consider two examples.

Example 1. We consider histosplines, i.e. splines for approximation of the density function of a random value by information of the frequencies of a density histogram on a given mesh $a=t_{0}<t_{1}<\cdots<t_{n}=b$ with additional boundary conditions.

We take $X=\mathbf{W}_{2}^{r}[a, b], Y=\mathbf{L}_{2}[a, b], T g=g^{(r)}, A=\left(k_{1}, \ldots, k_{n}\right)$ and $B=\left(l_{1}, l_{2}\right)$, where

$$
k_{i} g=\int_{t_{i-1}}^{t_{i}} g(t) d t, \quad i=1, \ldots, n, \quad l_{1} g=g(a), \quad l_{2} g=g(b) .
$$

A function $s_{1}$ is a spline from the space of histosplines $S(T, A)$ if and only if

$$
\begin{aligned}
s_{1}(t)= & \sum_{j=0}^{r-1} d_{j} t^{j}+\frac{(-1)^{r+1}}{(2 r) !} \sum_{i=1}^{n} \alpha_{i}\left(\left(t-t_{i}\right)_{+}^{2 r}-\left(t-t_{i-1}\right)_{+}^{2 r}\right), \\
& \sum_{i=1}^{n} \alpha_{i}\left(t_{i}^{j+1}-t_{i-1}^{j+1}\right)=0, \quad j=0, \ldots, r-1 .
\end{aligned}
$$

Space $S(T, B)$ consists of polynomials

$$
s_{2}(t)=\sum_{j=0}^{r-1} c_{j} t^{j}+\frac{(-1)^{r} \beta_{1}}{(2 r-1) !}(t-a)^{2 r-1}
$$

with coefficients which satisfy the equalities

$$
\beta_{1} a^{j}+\beta_{2} b^{j}=0, \quad j=0, \ldots, r-1 .
$$

By using both formulas we obtain the formula for spline $s \in S(T,(A, B))$ :

$$
s(t)=\sum_{j=0}^{r-1} \varrho_{j} t^{j}+\frac{(-1)^{r+1}}{(2 r) !} \sum_{i=1}^{n} \alpha_{i}\left(\left(t-t_{i}\right)_{+}^{2 r}-\left(t-t_{i-1}\right)_{+}^{2 r}\right)+\frac{(-1)^{r} \beta_{1}}{(2 r-1) !}(t-a)^{2 r-1}
$$

with the following conditions on the coefficients

$$
\beta_{1} a^{j}+\sum_{i=1}^{n} \frac{\alpha_{i}}{j+1}\left(t_{i}^{j+1}-t_{i-1}^{j+1}\right)+\beta_{2} b^{j}=0, \quad j=0,1, \ldots, r-1 .
$$


Example 2. We consider splines for approximation of a function $f$ by using data

$$
f_{i, j}=f^{(j)}\left(t_{i}\right), \quad j=0,1, i=1, \ldots, n,
$$

on a given mesh $a=t_{1}<t_{2}<\cdots<t_{n}=b$ under boundary conditions on

$$
\lambda_{1} f_{1,0}+\mu_{1} f_{1,1} \quad \text { and } \quad \lambda_{2} f_{n, 0}+\mu_{2} f_{n, 1}
$$

for given numbers $\lambda_{1}, \lambda_{2}, \mu_{1}, \mu_{2}$.

We take $X=\mathbf{W}_{2}^{r}[a, b], Y=\mathbf{L}_{2}[a, b], T g=g^{(r)}$, and

$$
\begin{aligned}
& k_{i} g=g\left(t_{i}\right), \quad k_{i+n} g=g^{\prime}\left(t_{i}\right), \quad i=1, \ldots, n, \\
& A g=\left(k_{2} g, \ldots, k_{n-1} g, k_{n+2} g, \ldots, k_{2 n-1} g\right), \\
& B g=\left(\lambda_{1} k_{1} g+\mu_{1} k_{n+1} g, \lambda_{2} k_{n} g+\mu_{2} k_{2 n} g\right) .
\end{aligned}
$$

A function $s_{1}$ is a spline from the space $S(T, A)$ of Hermite splines if and only if

$$
s_{1}(t)=\sum_{i=0}^{r-1} d_{i} t^{i}+\sum_{i=2}^{n-1}(-1)^{r}\left(\alpha_{i 0} \frac{\left(t-t_{i}\right)_{+}^{2 r-1}}{(2 r-1) !}-\alpha_{i 1} \frac{\left(t-t_{i}\right)_{+}^{2 r-2}}{(2 r-2) !}\right)
$$

with the conditions on the coefficients

$$
\sum_{i=2}^{n-1} \alpha_{i 0}=0, \quad \sum_{i=2}^{n-1} \alpha_{i 0} t_{i}^{j}+\sum_{i=2}^{n-1} \alpha_{i 1} j t_{i}^{j-1}=0, \quad j=1, \ldots, r-1 .
$$

Space $S(T, B)$ consists of polynomials

$$
s_{2}(t)=\sum_{i=0}^{r-1} c_{i} t^{i}+(-1)^{r} \beta_{1}\left(\lambda_{1} \frac{(t-a)^{2 r-1}}{(2 r-1) !}-\mu_{1} \frac{(t-a)^{2 r-2}}{(2 r-2) !}\right)
$$

with coefficients, which satisfy the equalities

$$
\begin{gathered}
\beta_{1}\left(\lambda_{1}+\mu_{1}\right)+\beta_{2}\left(\lambda_{2}+\mu_{2}\right)=0 \\
\beta_{1}\left(\lambda_{1} a^{j}+\mu_{1} j a^{j-1}\right)+\beta_{2}\left(\lambda_{2} b^{j}+\mu_{2} j b^{j-1}\right)=0, \quad j=1, \ldots, r-1 .
\end{gathered}
$$

By using both formulas we obtain the formula for spline $s \in S(T,(A, B))$ :

$$
\begin{aligned}
s(t)= & \sum_{i=0}^{r-1} \varrho_{i} t^{i}+\sum_{i=2}^{n-1}(-1)^{r}\left(\alpha_{i 0} \frac{\left(t-t_{i}\right)_{+}^{2 r-1}}{(2 r-1) !}-\alpha_{i 1} \frac{\left(t-t_{i}\right)_{+}^{2 r-2}}{(2 r-2) !}\right) \\
& +(-1)^{r} \beta_{1}\left(\lambda_{1} \frac{(t-a)^{2 r-1}}{(2 r-1) !}-\mu_{1} \frac{(t-a)^{2 r-2}}{(2 r-2) !}\right),
\end{aligned}
$$

with the following conditions on the coefficients

$$
\begin{gathered}
\sum_{i=2}^{n-1} \alpha_{i 0}+\beta_{1}\left(\lambda_{1}+\mu_{1}\right)+\beta_{2}\left(\lambda_{2}+\mu_{2}\right)=0 \\
\sum_{i=2}^{n-1} \alpha_{i 0} t_{i}^{j}+\sum_{i=2}^{n-1} \alpha_{i 1} j t_{i}^{j-1}+\beta_{1}\left(\lambda_{1} a^{j}+\mu_{1} j a^{j-1}\right)+\beta_{2}\left(\lambda_{2} b^{j}+\mu_{2} j b^{j-1}\right)=0, \\
j=1, \ldots, r-1 .
\end{gathered}
$$




\section{Special Cases of the Generalized Theorem}

Firstly, we show that Theorem 1 gives in some special cases the known results for Problems 1, 2, 3 and 4 .

By taking $A=B, \rho_{i}>0, i=1, \ldots, n$, and $C=\{\boldsymbol{v}\}$ in problem (3.1) we get the interpolating problem (2.1) and the well known theorem from the theory of splines (see, e.g., $[7,15,19]$ ).

Theorem 2. Under the assumption that $\operatorname{ker} T+\operatorname{ker} A$ is closed, a solution of (2.1) exists. An element $s \in X$ such that $A s=\boldsymbol{v}$ is a solution of this problem if and only if there exists a vector $\boldsymbol{\alpha} \in \mathbb{R}^{n}$ such that

$$
T^{*} T s=A^{*} \boldsymbol{\alpha} .
$$

For the case $A=B, \rho_{i}=0, i=1, \ldots, n$, our problem (3.1) is coincided with the problem on splines in convex set (2.2), and Theorem 1 gives the known result (see, e.g., [19]).

Theorem 3. Under the assumption that $\operatorname{ker} T+A^{-1}(C)$ is closed, a solution of problem (2.2) exists. An element $s \in A^{-1}(C)$ is a solution of this problem if and only if there exists a vector $\boldsymbol{\alpha} \in \mathbb{R}^{n}$ such that

$$
T^{*} T s=A^{*} \boldsymbol{\alpha}
$$

and $\langle\boldsymbol{\alpha}, \boldsymbol{c}-A s\rangle \geq 0$ for all $\boldsymbol{c} \in C$.

In the case when $A=B, C=\mathbb{R}^{n}, \rho_{i}>0, i=1, \ldots, n$, problem (3.1) could be rewritten as the smoothing problem with weights $(2.3)$.

Theorem 4. If $\operatorname{ker} T+\operatorname{ker} A$ is closed, then a solution of problem (2.3) exists. An element $s \in X$ is a solution of this problem if and only if there exists a vector $\boldsymbol{\alpha} \in \mathbb{R}^{n}$ such that

$$
T^{*} T s=A^{*} \boldsymbol{\alpha} \quad \text { and } \quad \boldsymbol{\alpha}+\mathbf{R}^{2}(A s-\boldsymbol{v})=\mathbf{0} .
$$

For the case $A=\left(k_{1}, \ldots, k_{n}\right), B=\left(l_{1}, \ldots, l_{m}\right)$, where functionals $k_{1}, \ldots, k_{n}$, $l_{1}, \ldots, l_{m}$ are linear independent, $\rho_{i}>0, i=1, \ldots, n$, and $C=\{\boldsymbol{u}\}$, from problem (3.1) we obtain the classical mixed interpolating-smoothing problem (2.4), for which the following theorem holds.

Theorem 5. If $\operatorname{ker} T+\operatorname{ker} A$ is closed, then a solution of problem (2.4) exists. An element $s \in X$ such that $B s=\boldsymbol{u}$ is a solution of this problem if and only if there exist vectors $\boldsymbol{\alpha} \in \mathbb{R}^{n}$ and $\boldsymbol{\beta} \in \mathbb{R}^{m}$ such that

$$
T^{*} T s=A^{*} \boldsymbol{\alpha}+B^{*} \boldsymbol{\beta} \quad \text { and } \quad \boldsymbol{\alpha}+\mathbf{R}^{2}(A s-\boldsymbol{v})=\mathbf{0} .
$$

On the other hand, from Theorem 1 we can obtain also new results for other special cases. For example, if we take $A=\mathbf{M} B$, where $\mathbf{M}$ is a given matrix $n \times m$, then we can consider problem

$$
\|T x\|^{2}+\|\mathbf{R}(\mathbf{M} B x-\boldsymbol{v})\|^{2} \longrightarrow \min _{x \in B^{-1}(C)}
$$

and obtain the following theorem. 
Theorem 6. Under the assumption that

$\operatorname{ker} T+\operatorname{ker} \mathbf{R M} B \quad$ and $\operatorname{ker} T \cap \operatorname{ker} \mathbf{R M} B+B^{-1}(C)$ are closed,

a solution of problem (4.1) exists. An element $s \in B^{-1}(C)$ is a solution of this problem if and only if there exists a vector $\boldsymbol{\lambda} \in \mathbb{R}^{m}$ such that

$$
T^{*} T s=B^{*} \boldsymbol{\lambda}
$$

and for all $c \in C$

$$
\left\langle\boldsymbol{\lambda}+\mathbf{M}^{T} \mathbf{R}^{2}(\mathbf{M} B s-\boldsymbol{v}), \boldsymbol{c}-B s\right\rangle \geq 0 .
$$

From this theorem it follows that a solution of $(4.1)$ is from $S(T, B)$.

Proof. The existence of a solution follows from Theorem 1. By it we also have that there exist vectors $\boldsymbol{\alpha} \in \mathbb{R}^{n}$ and $\boldsymbol{\beta} \in \mathbb{R}^{m}$ such that

$$
T^{*} T s=A^{*} \boldsymbol{\alpha}+B^{*} \boldsymbol{\beta}
$$

and

$$
\left\langle\boldsymbol{\alpha}+\mathbf{R}^{2}(A s-\boldsymbol{v}), \boldsymbol{d}-A s\right\rangle+\langle\boldsymbol{\beta}, \boldsymbol{c}-B s\rangle \geq 0
$$

for all $\boldsymbol{c} \in C$ and for all $\boldsymbol{d} \in A\left(B^{-1}(\boldsymbol{c})\right)$.

Let us consider how these conditions could be rewritten in our case. We start with

$$
\begin{aligned}
\left\langle T^{*} T s, x\right\rangle=\left\langle A^{*} \boldsymbol{\alpha}, x\right\rangle+\left\langle B^{*} \boldsymbol{\beta}, x\right\rangle & =\langle\boldsymbol{\alpha}, A x\rangle+\langle\boldsymbol{\beta}, B x\rangle \\
& =\langle\boldsymbol{\alpha}, \mathbf{M} B x\rangle+\langle\boldsymbol{\beta}, B x\rangle=\left\langle\mathbf{M}^{T} \boldsymbol{\alpha}+\boldsymbol{\beta}, B x\right\rangle .
\end{aligned}
$$

We denote $\boldsymbol{\lambda}=\mathbf{M}^{T} \boldsymbol{\alpha}+\boldsymbol{\beta}$ and obtain that $T^{*} T s=B^{*} \boldsymbol{\lambda}$.

In our case $\operatorname{ker} B \subset \operatorname{ker} A$. It means that for a vector $c \in C$ we have $A\left(B^{-1}(\boldsymbol{c})\right)=A\left(x_{\boldsymbol{c}}+\operatorname{ker} B\right)=A x_{\boldsymbol{c}}$, where $x_{\boldsymbol{c}}$ is an element from $B^{-1}(\boldsymbol{c})$. If $A x=\mathbf{M} B x$, then $\boldsymbol{d}=A x_{\boldsymbol{c}}=\mathbf{M} B x_{\boldsymbol{c}}=\mathbf{M} \boldsymbol{c}$ and for all $\boldsymbol{c} \in \mathbb{R}^{m}$ and $\boldsymbol{d}=\mathbf{M} \boldsymbol{c}$ we have

$$
\begin{aligned}
0 & \leq\left\langle\boldsymbol{\alpha}+\mathbf{R}^{2}(A s-\boldsymbol{v}), \mathbf{M} \boldsymbol{c}-\mathbf{M} B s\right\rangle+\langle\boldsymbol{\beta}, \boldsymbol{c}-B s\rangle \\
& =\left\langle\mathbf{M}^{T} \boldsymbol{\alpha}+\mathbf{M}^{T} \mathbf{R}^{2}(A s-\boldsymbol{v}), \boldsymbol{c}-B s\right\rangle+\langle\boldsymbol{\beta}, \boldsymbol{c}-B s\rangle \\
& =\left\langle\mathbf{M}^{T} \boldsymbol{\alpha}+\boldsymbol{\beta}+\mathbf{M}^{T} \mathbf{R}^{2}(A s-\boldsymbol{v}), \boldsymbol{c}-B s\right\rangle .
\end{aligned}
$$

Therefore for all $c \in C$

$$
\left\langle\boldsymbol{\lambda}+\mathbf{M}^{T} \mathbf{R}^{2}(\mathbf{M} B s-\boldsymbol{v}), \boldsymbol{c}-B s\right\rangle \geq 0 .
$$

Now we consider (4.1) for $C=\Pi_{i=1}^{m}\left[a_{i} ; b_{i}\right] \subset \mathbb{R}^{m}$ :

$$
\|T x\|^{2}+\|\mathbf{R}(\mathbf{M} B x-\boldsymbol{v})\|^{2} \longrightarrow \min _{a_{i} \leq(B x)_{i} \leq b_{i}, i=1, \ldots, m} .
$$


Theorem 7. Under the assumption that

$\operatorname{ker} T+\operatorname{ker} \mathbf{R M} B$ and $\operatorname{ker} T \cap \operatorname{ker} \mathbf{R M} B+B^{-1}(C)$ are closed, a solution of problem (4.2) exists. An element $s \in B^{-1}(C)$ is a solution of this problem if and only if there exist a vector $\boldsymbol{\lambda} \in \mathbb{R}^{m}$ such that

$$
T^{*} T s=B^{*} \boldsymbol{\lambda}
$$

and

- $\left(\boldsymbol{\lambda}+\mathbf{M}^{T} \mathbf{R}^{2}(\mathbf{M} B s-\boldsymbol{v})\right)_{i} \leq 0$ if $(B s)_{i}=b_{i}$,

- $\left(\boldsymbol{\lambda}+\mathbf{M}^{T} \mathbf{R}^{2}(\mathbf{M} B s-\boldsymbol{v})\right)_{i} \geq 0$ if $(B s)_{i}=a_{i}$,

- $\left(\boldsymbol{\lambda}+\mathbf{M}^{T} \mathbf{R}^{2}(\mathbf{M} B s-\boldsymbol{v})\right)_{i}=0$ if $(B s)_{i} \in\left(a_{i} ; b_{i}\right), i=1, \ldots, m$.

Proof. To prove the result on the signs of the components of vector

$$
\boldsymbol{\lambda}+\mathbf{M}^{T} \mathbf{R}^{2}(\mathbf{M} B s-\boldsymbol{v}),
$$

we use the inequality

$$
\left\langle\boldsymbol{\lambda}+\mathbf{M}^{T} \mathbf{R}^{2}(\mathbf{M} B s-\boldsymbol{v}), \boldsymbol{c}-B s\right\rangle \geq 0,
$$

which is true for all $c \in C$.

If $(B s)_{i}=b_{i}$ for some $i$, then we can take $c_{j}=(B s)_{j}, j=1, \ldots, n, j \neq i$, and $c_{i} \in\left(a_{i} ; b_{i}\right)$, and obtain $\left(\boldsymbol{\lambda}+\mathbf{M}^{T} \mathbf{R}^{2}(\mathbf{M} B s-\boldsymbol{v})\right)_{i} \leq 0$ because of $c_{i}-b_{i} \leq 0$. By analogy for the case $(B s)_{i}=a_{i}$ we can obtain $\left(\boldsymbol{\lambda}+\mathbf{M}^{T} \mathbf{R}^{2}(\mathbf{M} B s-\boldsymbol{v})\right)_{i} \geq 0$ because of $c_{i}-a_{i} \geq 0$.

If $(B s)_{i} \in\left(a_{i} ; b_{i}\right)$ for some $i$, then

1) we can take $c_{j}=(B s)_{j}, j=1, \ldots, n, j \neq i$, and $c_{i}=b_{i}$, and obtain that $\left(\boldsymbol{\lambda}+\mathbf{M}^{T} \mathbf{R}^{2}(\mathbf{M} B s-\boldsymbol{v})\right)_{i} \geq 0$ because of $c_{i}-(B s)_{i}>0 ;$

2) we can take $c_{j}=(B s)_{j}, j=1, \ldots, n, j \neq i$, and $c_{i}=a_{i}$, and obtain that $\left(\boldsymbol{\lambda}+\mathbf{M}^{T} \mathbf{R}^{2}(\mathbf{M} B s-\boldsymbol{v})\right)_{i} \leq 0$ because of $c_{i}-(B s)_{i}<0$.

Therefore in this case $\left(\boldsymbol{\lambda}+\mathbf{M}^{T} \mathbf{R}^{2}(\mathbf{M} B s-\boldsymbol{v})\right)_{i}=0$.

Now let us take $B=\mathbf{M} A$, where $\mathbf{M}$ is a given matrix $m \times n$, and consider the following problem

$$
\|T x\|^{2}+\|\mathbf{R}(A x-\boldsymbol{v})\|^{2} \longrightarrow \min _{x \in(\mathbf{M} A)^{-1}(C)} .
$$

Theorem 8. Under the assumption that

$\operatorname{ker} T+\operatorname{ker} \mathbf{R} A$ and $\operatorname{ker} T \cap \operatorname{ker} \mathbf{R} A+(\mathbf{M} A)^{-1}(C)$ are closed,

a solution of problem (4.3) exists. An element $s \in(\mathbf{M} A)^{-1}(C)$ is a solution of this problem if and only if there exists a vector $\boldsymbol{\lambda} \in \mathbb{R}^{n}$ such that

$$
T^{*} T s=A^{*} \boldsymbol{\lambda}
$$

and for all $\boldsymbol{d} \in \mathbb{R}^{n}$, such that $\mathbf{M d} \in C$, it holds

$$
\left\langle\boldsymbol{\lambda}+\mathbf{R}^{2}(A s-\boldsymbol{v}), \boldsymbol{d}-A s\right\rangle \geq 0 .
$$


From this theorem it follows that a solution of (4.3) is from $S(T, A)$.

Proof. We apply the technique which is analogous to the technique used in the proof of Theorem 6 . For a solution $s$ of problem (4.3) and for $x \in X$ we rewrite

$$
\begin{aligned}
\left\langle T^{*} T s, x\right\rangle & =\left\langle A^{*} \boldsymbol{\alpha}, x\right\rangle+\left\langle B^{*} \boldsymbol{\beta}, x\right\rangle=\langle\boldsymbol{\alpha}, A x\rangle+\langle\boldsymbol{\beta}, B x\rangle \\
& =\langle\boldsymbol{\alpha}, A x\rangle+\langle\boldsymbol{\beta}, \mathbf{M} A x\rangle=\left\langle\boldsymbol{\alpha}+\mathbf{M}^{T} \boldsymbol{\beta}, A x\right\rangle .
\end{aligned}
$$

Now we denote $\boldsymbol{\lambda}=\boldsymbol{\alpha}+\mathbf{M}^{T} \boldsymbol{\beta}$ and obtain that $T^{*} T s=A^{*} \boldsymbol{\lambda}$.

For a vector $\boldsymbol{c} \in C$ we have $A\left(B^{-1}(\boldsymbol{c})\right)=A x_{\boldsymbol{c}}+A(\operatorname{Ker} B)$, where $x_{\boldsymbol{c}}$ is an element from $B^{-1}(\boldsymbol{c})$. In our case $\operatorname{ker} A \subset \operatorname{ker} B$, therefore $\boldsymbol{d} \in A\left(B^{-1}(\boldsymbol{c})\right)$ for some vector $\boldsymbol{c} \in C$ if and only if $\mathbf{M} \boldsymbol{d} \in C$. By taking $\boldsymbol{d} \in \mathbb{R}^{n}$, such that $\mathbf{M} \boldsymbol{d} \in C$, we obtain

$$
\begin{aligned}
0 & \leq\left\langle\boldsymbol{\alpha}+\mathbf{R}^{2}(A s-\boldsymbol{v}), \boldsymbol{d}-A s\right\rangle+\langle\boldsymbol{\beta}, \mathbf{M} \boldsymbol{d}-\mathbf{M} A s\rangle \\
& =\left\langle\boldsymbol{\alpha}+\mathbf{M}^{T} \boldsymbol{\beta}+\mathbf{R}^{2}(A s-\boldsymbol{v}), \boldsymbol{d}-A s\right\rangle .
\end{aligned}
$$

It means that $\left\langle\boldsymbol{\lambda}+\mathbf{R}^{2}(A s-\boldsymbol{v}), \boldsymbol{d}-A s\right\rangle \geq 0$.

\section{Examples}

To show how our result works we consider some simple numerical examples. Let us start with the problem of approximation of the density function of a random value given by a histogram $F=\left\{f_{1}, \ldots, f_{n}\right\}$. Here $f_{i}$ is a frequency for the interval $\left[t_{i-1}, t_{i}\right]$ (we denote $h_{i}=t_{i}-t_{i-1}$ ), $i=1, \ldots, n$, of a given mesh $a=t_{0}<t_{1}<\cdots<t_{n}=b$ of the interval $[a, b]$. We consider the following smoothing problem with the additional boundary conditions:

$$
\int_{a}^{b}\left(x^{\prime \prime}(t)\right)^{2} d t+\sum_{i=1}^{n} \rho_{i}\left(\int_{t_{i-1}}^{t_{i}} x(t) d t-f_{i} h_{i}\right)^{2} \longrightarrow \min _{\substack{x(t) \in \mathbf{W}_{2}^{2}[a, b], x(a)=0, x(b)=0}} .
$$

The solution $s$ of problem (5.1) belongs to the space of mixed splines (see Example 1) and it could be written by formula (3.7)-(3.8) with $r=2$. Spline $s$ could be obtained taking into account Theorem 5 as the solution of the following system of linear equations:

$$
\begin{aligned}
& \alpha_{i}+\rho_{i} \int_{t_{i-1}}^{t_{i}} s(t) d t=\rho_{i} f_{i} h_{i}, \quad i=1, \ldots, n, \\
& s(a)=0, \quad s(b)=0 .
\end{aligned}
$$

The solution of this system in the case

$$
\begin{aligned}
& f_{1}=\frac{1}{58}, \quad f_{2}=\frac{2}{58}, \quad f_{3}=\frac{6}{29}, \quad f_{4}=\frac{1}{58}, \quad f_{5}=\frac{15}{58}, f_{6}=\frac{9}{58}, f_{7}=\frac{2}{58} \\
& f_{8}=\frac{1}{58}, \quad f_{9}=\frac{9}{58}, \quad f_{10}=\frac{5}{58}, \quad f_{11}=\frac{1}{58} \\
& a=0, \quad b=11, \quad h_{i}=1, \quad \rho_{i}=10^{3}, \quad i=1, \ldots, 11
\end{aligned}
$$


gives us

$$
\begin{aligned}
s(t) & =0,091968 t+0,142064 t^{4}-0.353077(t-1)_{+}^{4}+0,505788(t-2)_{+}^{4} \\
& -0,614901(t-3)_{+}^{4}+0,570003(t-4)_{+}^{4}-0,383120(t-5)_{+}^{4}+0.209387(t-6)_{+}^{4} \\
& -0,170771(t-7)_{+}^{4}+0,193087(t-8)_{+}^{4}-0,153887(t-9)_{+}^{4}+0,073022(t-10)_{+}^{4} \\
& -0,017595(t-11)_{+}^{4}-0,242297 t^{3} .
\end{aligned}
$$

The graph of $s$ is shown in Figure 1 .

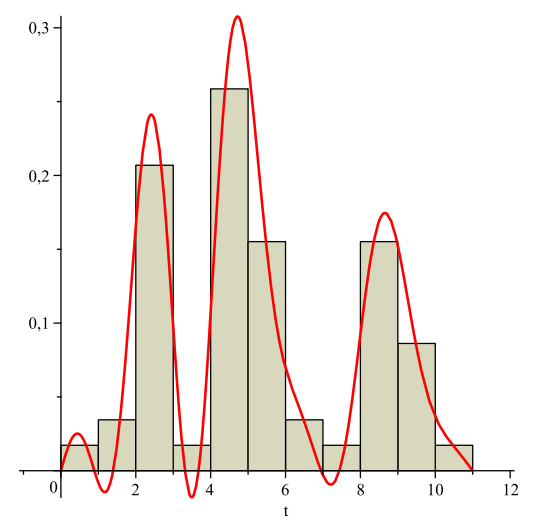

Figure 1. The solution of problem (5.1).

It is easy to see that this spline could not be considered as a solution of the problem of approximation of the density function because of negativity of it in some subintervals of $[a, b]$. We cannot solve problem (5.1) with the additional condition $x(t) \geq 0$ and we suggest to consider instead of (5.1) the following problem with nonnegativity conditions at some control points $\tau_{j} \in(a ; b), j=$ $3, \ldots, m$ :

$$
\int_{a}^{b}\left(x^{\prime \prime}(t)\right)^{2} d t+\sum_{i=1}^{n} \rho_{i}\left(\int_{t_{i-1}}^{t_{i}} x(t) d t-f_{i} h_{i}\right)^{2} \longrightarrow \min _{\begin{array}{c}
x(t) \in \mathbf{W}_{2}^{2}[a, b], \\
x(a)=0, x(b)=0, \\
x\left(\tau_{j}\right) \geq 0, j=3, \ldots, m
\end{array}} .
$$

By Theorem 1 the solution of (5.2) is a spline $s$ from $S(T,(A, B))$, where

$$
\begin{gathered}
k_{i} x=\int_{t_{i-1}}^{t_{i}} x(t) d t, \quad i=1, \ldots, n, \\
l_{1} x=x(a), \quad l_{2} x=x(b), \quad l_{j} x=x\left(\tau_{j}\right), \quad j=3, \ldots, m .
\end{gathered}
$$

It means, that spline $s$ is in the form

$$
\begin{aligned}
s(t)= & \sum_{j=0}^{r-1} \varrho_{j} t^{j}+\frac{(-1)^{r+1}}{(2 r) !} \sum_{i=1}^{n} \alpha_{i}\left(\left(t-t_{i}\right)_{+}^{2 r}-\left(t-t_{i-1}\right)_{+}^{2 r}\right)+ \\
& +\frac{(-1)^{r} \beta_{1}}{(2 r-1) !}(t-a)^{2 r-1}+\sum_{j=3}^{m} \frac{(-1)^{r} \beta_{j}}{(2 r-1) !}\left(t-\tau_{j}\right)^{2 r-1}
\end{aligned}
$$


with the following conditions on the coefficients:

$$
\beta_{1} a^{p}+\sum_{i=1}^{n} \alpha_{i}\left(t_{i}^{p+1}-t_{i-1}^{p+1}\right)+\beta_{2} b^{p}+\sum_{j=3}^{m} \beta_{j}\left(\tau_{j}\right)^{p}=0, \quad p=0,1, \ldots, r-1 .
$$

We take $C=\left\{\left(0,0, u_{3}, \ldots, u_{m}\right) \mid u_{j} \geq 0, j=3, \ldots, m\right\}$. Now by using Theorem 8 we can obtain that an element $s \in B^{-1}(C)$ is a solution of (5.2) if and only if

- $\alpha_{i}+\rho_{i}\left(k_{i} s-f_{i} v_{i}\right)=0$ for all $i=1, \ldots, n$;

- $\beta_{j} \geq 0$ if $l_{j} s=0, j=3, \ldots, m$;

- $\beta_{j}=0$ if $l_{j} s>0, j=3, \ldots, m$.

To obtain this result we consider $\tilde{A} x=\left(k_{1} x, \ldots, k_{n} x, l_{1} x, \ldots, l_{m} x\right), \tilde{B}=\mathbf{M} \tilde{A}$ and $\rho_{n+j}=0, j=1, \ldots, m$, where $\mathbf{M}$ is $m \times(n+m)$ matrix with the components which correspond to the conditions of (5.2). The first $n$ columns of $\mathbf{M}$ consist only of zeroes. By Theorem 8 the solution of $(5.2)$ is a spline $s$ from $S(T, \tilde{A})$, i.e. there exists a vector $\boldsymbol{\lambda}=\left(\alpha_{1}, \ldots, \alpha_{n}, \beta_{1}, \ldots, \beta_{m}\right)$ such that

$$
T^{*} T s=\tilde{A}^{*} \boldsymbol{\lambda}
$$

and for all $\boldsymbol{d} \in \mathbb{R}^{n+m}$, such that $\mathbf{M} \boldsymbol{d} \in C$, it holds

$$
\left\langle\boldsymbol{\lambda}+\mathbf{R}^{2}(\tilde{A} s-\boldsymbol{v}), \boldsymbol{d}-\tilde{A} s\right\rangle \geq 0
$$

By taking $d_{i} \neq(\tilde{A} s)_{i}$ for some $i \in\{1, \ldots, n\}$ and $d_{j}=(\tilde{A} s)_{j}$ for all $j \neq i$ we obtain $\alpha_{i}+\rho_{i}\left(k_{i} s-f_{i} h_{i}\right)=0$, since we can take $d_{i}>(\tilde{A} s)_{i}$ and $d_{i}<(\tilde{A} s)_{i}$.

In the case, when $l_{j} s=0$ for some $j \in\{3, \ldots, m\}$, by taking $d_{n+j}>0$ and $d_{i}=(\tilde{A} s)_{i}$ for all $i \neq n+j$ we obtain $\beta_{j} \geq 0$.

In the case, when $l_{j} s>0$ for some $j \in\{3, \ldots, m\}$, by taking $d_{n+j} \neq l_{j} s$ and $d_{i}=(\tilde{A} s)_{i}$ for all $i \neq n+j$ we obtain $\beta_{j}=0$, since we can take $d_{n+j}>l_{j} s$ and $d_{n+j}<l_{j} s$.

The solution of $(5.2)$ with control points $\tau_{3}=1.17, \tau_{4}=3.5, \tau_{5}=7.2$ is

$$
\begin{aligned}
s(t)= & 0,07369 t+0,087607 t^{4}-0.402177(t-1)_{+}^{4}+0,660935(t-2)_{+}^{4} \\
& -1,011544(t-3)_{+}^{4}+0.941866(t-4)_{+}^{4}-0,410172(t-5)_{+}^{4}+0.192637 \\
& \times(t-6)_{+}^{4}-0,214509(t-7)_{+}^{4}+0,269641(t-8)_{+}^{4}-0,174781(t-9)_{+}^{4} \\
& +0,074955(t-10)_{+}^{4}-0,014459(t-11)_{+}^{4}-0,155669 t^{3} \\
& +0,450560(t-1.17)_{+}^{3}+1.176316(t-3.5)_{+}^{3}+0,250122(t-7.2)_{+}^{3} .
\end{aligned}
$$

The graph of $s$ is shown in Figure 2 .

Let us note that one additional condition is very important in the case of approximation of density functions as the property of density histograms (the area under them is equal to 1 ). That is why we insert this additional condition 


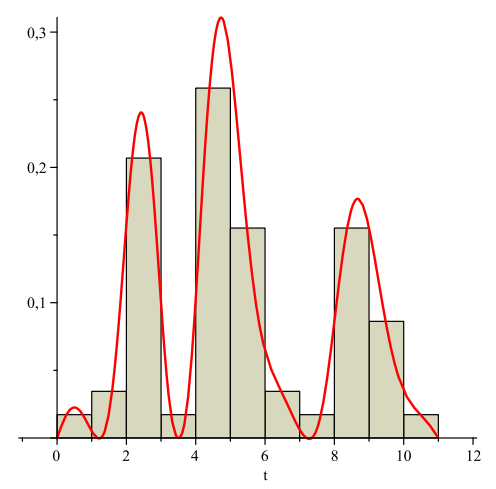

Figure 2. The solution of problem (5.2).

into (5.2) and consider the following problem:

$$
\int_{a}^{b}\left(x^{\prime \prime}(t)\right)^{2} d t+\sum_{i=1}^{n} \rho_{i}\left(\int_{t_{i-1}}^{t_{i}} x(t) d t-f_{i} h_{i}\right)^{2} \longrightarrow \min _{\begin{array}{c}
x(t) \in \mathbf{W}_{2}^{2}[a, b] \\
\int_{a}^{b} x(t) d t=1 \\
x(a)=0, x(b)=0 \\
x\left(\tau_{j}\right) \geq 0, j=3, \ldots, m
\end{array}}
$$

Therefore now we take

$$
\begin{gathered}
k_{i} x=\int_{t_{i-1}}^{t_{i}} x(t) d t, \quad i=1, \ldots, n, \quad l_{0} x=\sum_{i=1}^{n} k_{i} x, \\
l_{1} x=x(a), \quad l_{2} x=x(b), \quad l_{j} x=x\left(\tau_{j}\right), \quad j=3, \ldots, m .
\end{gathered}
$$

For this case in contrast to (5.2) we have $l_{0}$ which is linear dependent on all $k_{i}$, $i=1, \ldots, n$. To solve $(5.5)$ we use Theorem 8 with

$$
\tilde{A} x=\left(k_{1} x, \ldots, k_{n} x, l_{1} x, \ldots, l_{m} x\right), \quad \tilde{B}=\mathbf{M} \tilde{A}
$$

and $\rho_{n+j}=0, j=1, \ldots, m$, where $\mathbf{M}$ is $(m+1) \times(n+m)$ matrix with the components which correspond to the conditions of $(5.5)$,

$$
C=\left\{\left(1,0,0, u_{3}, \ldots, u_{m}\right) \mid u_{j} \geq 0, j=3, \ldots, m\right\} .
$$

By Theorem 8 the solution of $(5.5)$ is a spline $s$ from $S(T, \tilde{A})$, i.e. there exists a vector $\boldsymbol{\lambda}=\left(\alpha_{1}, \ldots, \alpha_{n}, \beta_{1}, \ldots, \beta_{m}\right)$ such that

$$
T^{*} T s=\tilde{A}^{*} \boldsymbol{\lambda}
$$

It means that the formula for $s$ is the same as in the previous example (see (5.3)-(5.4)). By applying the technique which is analogous to the technique used in the previous case from Theorem 8 we could obtain that an element $s \in(\mathbf{M} \tilde{A})^{-1}(C)$ is a solution of (5.5) if and only if

- $\left(\alpha_{i}-\frac{1}{n} \sum_{j=1}^{n} \alpha_{j}\right)+\rho_{i}\left(k_{i} s-f_{i} h_{i}\right)=0$ for all $i=1, \ldots, n$; 
- $\beta_{j} \geq 0$ if $l_{j} s=0, j=3, \ldots, m$;

- $\beta_{j}=0$ if $l_{j} s>0, j=3, \ldots, m$.

The solution of problem (5.5) with the same data as in (5.2) is spline

$$
\begin{aligned}
s(t)= & 0.068636 t+0.081903 t^{4}-0.404141(t-1)_{+}^{4}+0.671881(t-2)_{+}^{4} \\
& -1.034427(t-3)_{+}^{4}+0.962872(t-4)_{+}^{4}-0.411178(t-5)_{+}^{4}+0.190207 \\
& \times(t-6)_{+}^{4}-0.218965(t-7)_{+}^{4}+0.277659(t-8)_{+}^{4}-0.176466(t-9)_{+}^{4} \\
& +0.074978(t-10)_{+}^{4}-0.014322(t-11)_{+}^{4}-0.145754 t^{3}+0.487652 \\
& \times(t-1.17)_{+}^{3}+1.242923(t-3.5)_{+}^{3}+0.277414(t-7.2)_{+}^{3} .
\end{aligned}
$$

Its graph is very similar to the graph from Figure 2.

\section{Conclusion}

In this paper we suggested a new generalization of smoothing problems in abstract Hilbert spaces and proved the theorem on the existence and characterization of a solution of the generalized problem. It was shown how the theorem gives already known theorems in special cases as well as some new results. The obtained results were illustrated with numerical examples, which motivate the generalization presented here. Last numerical examples could not be solved without the obtained theorem; this clarifies the importance of the result. Future work will mainly focus on investigation of new special cases of the generalized problem by specifying the spaces and operators, including the framework of multivariate variational splines.

\section{References}

[1] P.M. Anselone and P.J. Laurent. A general method for the construction of interpolating or smoothing spline-functions. Numer. Math., 12:66-82, 1968. http://dx.doi.org/10.1007/BF02170998.

[2] R. Arcangeli, M.C. Lopez de Silanes and J.J. Torrens. Multidimensional Minimizing Splines. Kluwer Academic Publishers, Boston, 2004.

[3] S. Asmuss and N. Budkina. Splines in convex sets under constraints of twosided inequality type in a hiperplane. Math. Model. Anal., 13(4):461-470, 2008. http://dx.doi.org/10.3846/1392-6292.2008.13.461-470.

[4] S. Asmuss and N. Budkina. On smoothing problems with one additional equality condition. Math. Model. Anal., 14(2):159-168, 2009. http://dx.doi.org/10.3846/1392-6292.2009.14.159-168.

[5] M. Attea. Generalisation de la definition et des properietes des. C. R. Acad. Sci., 260:3550-3553, 1965.

[6] M. Attea. Fonctions spline definies sur un ensemble convexe. Numer. Math., 12:192-210, 1968. http://dx.doi.org/10.1007/BF02162912.

[7] A.Yu. Bezhaev and V.A. Vasilenko. Variational Theory of Splines. Kluwer Academic/Plenum Publishers, New York, 2001. 
[8] J.C. Holladay. Smoothest curve approximation. Math. Tables Aids Computation, 11:233-243, 1957. http://dx.doi.org/10.2307/2001941.

[9] A. Kouibia and M. Pasadas. Smoothing variational splines. Appl. Math. Lett., 13:71-75, 2000. http://dx.doi.org/10.1016/S0893-9659(99)00167-6.

[10] A. Kouibia and M. Pasadas. Approximation of curves by fairness splines with tangent conditions. J. Comput. Appl. Math., 142:357-366, 2002.

http://dx.doi.org/10.1016/S0377-0427(01)00428-9.

[11] A. Kouibia and M. Pasadas. Approximation by interpolating variational splines. J. Comput. Appl. Math., 218:342-349, 2008. http://dx.doi.org/10.1016/j.cam.2007.02.042.

[12] A. Kouibia and M. Pasadas. Approximation by smoothing variational vector splines for noisy data. J. Comput. Appl. Math., 211:213-222, 2008. http://dx.doi.org/10.1016/j.cam.2006.11.016.

[13] A. Kouibia and M. Pasadas. Bivariate variational splines with monotonicity constraints. Math. Comput. Simulation, 77:228-236, 2008. http://dx.doi.org/10.1016/j.matcom.2007.08.004.

[14] A. Kouibia and M. Pasadas. An approximation problem of noisy data by cubic and bicubic splines. Appl. Math. Model., 36:4135-4145, 2012. http://dx.doi.org/10.1016/j.apm.2011.11.043.

[15] P.J. Laurent. Approximation et optimisation. Hermann, Paris, 1972.

[16] E. Leetma. A method for solving classical smoothing problems with obstacles. Proc. Est. Acad. Sci., 58(4):197-204, 2009. http://dx.doi.org/10.3176/proc.2009.4.01.

[17] E. Leetma and P. Oja. A method of adding-removing knots for solving smoothing problems with obstacles. European J. Oper. Res., 194(1):28-38, 2009. http://dx.doi.org/10.1016/j.ejor.2007.12.020.

[18] E. Leetma and P. Oja. Existence of the solution of classical smoothing problems with obstacles and weights. Math. Model. Anal., 17(1):58-64, 2012. http://dx.doi.org/10.3846/13926292.2012.644869.

[19] V. Vershinin, Yu. Zavyalov and N. Pavlov. Extremal Properties of Splines and the Smoothing Problem. Nauka, Novosibirsk, 1988. 